\title{
Organization and Classification of Nanotechnology: A Pathway to the Standardization of Nanotechnology
}

\author{
Edilson Gomes de Lima
}

Institute of Engineering in Nanoscience and Nanotechnology, Australia

Copyright $(2017$ by authors, all rights reserved. Authors agree that this article remains permanently open access under the terms of the Creative Commons Attribution License 4.0 International License

\begin{abstract}
The purpose of this paper is to create a procedure for classifying and organizing nanotechnology. In addition to presenting the importance of diagrams, tables and scientific, engineering, analytical diagrams for technical consultation in nanotechnology. Considering a question such as: what is the percentage of nanotechnology in your product, service or process? Consider answering this question by consulting a standardized set of diagrams, at the level of Mollier's diagram thermodynamics. A set of diagrams have already been considered and contemplate various results of analytical tests, and characterizations of specific nanosciences for use in nanotechnology. No need for costly analytical or validation testing. This is the initial proposal of this paper. We have heard in many cases experts in their fields, especially in chemistry, to say that nanotechnology is an integral technique from chemistry. Your interests can be pulled into this new science. Although this science was born in physics [1]. One of the intentions of this paper is the search for the emancipation of the nanotechnology through standardization norms, through diagrams, as in engineering [8]. Because, nanoscience and nanotechnology are something of their own, a new discipline with their own ranges of jobs. Therefore, nanotechnology needs to emancipate itself from the classical sciences, and will only be able to achieve this stage after standardization, normalization and with its own scientific, engineering diagrams and technical standards. And this paper will present the way for nanotechnology to finally have its emancipation from the classical sciences.
\end{abstract}

Keywords Organization, Classification, Emancipation, Crowdsourcing and Standardization of Nanotechnology

"The first step to organizing is to sort and list neatly. So, reaching a ranking of technological complexity. Thus, we can conclude that after the organization of complexity we have an effective and practical simplicity ".

Edilson Gomes de Lima

\section{Presentation}

From the expansion of wealth to the generation of jobs, many projections are already being raised with concrete data and information. Here is importance the use of data mining techniques, including statistics, artificial intelligence and machine learning. Following the steps, data sources, data exploration gathering, modeling and deploying models [3]. The term nanoscience \& nanotechnology has economic power and change in society broadly. $\mathrm{N} \& \mathrm{~N}$ presents itself as an additional study steam at academies worldwide, in which we observe any traditional discipline adding the term nano in its studies. The interesting thing is that starting from the condensation of consolidated traditional knowledge, many innovations can enter the industrial sector generating products based on nanotechnology. An interface between the nanometer and the macrometric dimension is something that involves a combination of disciplines. As an interface between antibodies, electrical reading at $\mathrm{eV}$ electron-Volts, interpretation in complex systems. It is not as clear as a dimensional reduction interface between gears, e.g. from $1 \mathrm{~mm}$ to $1 \mathrm{~cm}$ and to $10 \mathrm{~cm}$, gradually. In computing the interface between human senses and bytes is given by electricity and mechanical-electronic devices. In the case of nanotechnology, we are at the heart of the matter, that is, by the principle of Heisenberg's uncertainty, the state of a given system will be disturbed if measured. The very interference of photons from the light to measure something would already cause interference in the measurement. Therefore, analytical apparatuses precisely calibrate in the nanometric world, are examples for interface techniques. To work with I.S international system, with units from (Siemens) to (eV) and others. Nanoscience's are already added to a great deal of information. We can say that nanoscience today is a mature science and very modern. However, the way of translating these concepts into nanoscience, with the classical disciplines, is a work without limits. That is, a highly creative engineering technical work. The work of engineering and technical creativity is precisely to create what does not exist, and to make it exist through science. In 
other words, moving away from the conceptual field of nanoscience, we enter in a more practical field, translating and trying to interconnect the subatomic world to our macroscopic world. And this through technological interfaces that do not yet exist, and need to be created. To this we call innovation and technology, that is, nanotechnology which are products and services with nanoscience aggregated in a practical way. And in the meantime, it is important to have a classification to define the degree or level of nanoscience added to new nanotechnology products and services. The objective desired by the object of investigation is not always achieved. Sometimes it is important to divert the focus to areas that do not really have any connection with the research object to achieve the desired goal. One example is the proposal to create a ranking for nanotechnology. To achieve full organization and functional classification, we can include techniques from other disciplines. That is, by making use of business intelligence techniques, analytical computing techniques such as specific dashboards for nanotechnology. As we can see in the case example that does not exactly match nanotechnology, but serves as reference [10] to be followed in the matter of data and information organization. And this classification is important because several factors. As for developers to visualize the following paths, investors, researchers and scientists direct the focus without wasting time. A nanotechnology rating is important because this technoscience is huge, and separating specialized action fields is important. A fragmentation of specializations that unite to achieve high level in the ranking. Therefore, one should not confuse sectorization with classification, because sectorization ends with freedom. The proposed classification has the sole purpose of organizing and classifying specific areas of the nanoscience and nanotechnology. For a first classification model nanotechnology.

\section{Introduction}

For a technological classification to organize, encourage, direct, improve and professionalize the nanoscience and nanotechnology sector. As an example, to direct the actions of promotional agents, scientists, students, industrialists, investors and researchers. That is, with a rating / ranking, these can direct their focus of action, without wasting time and focus, targeting exactly in a niche. Initially, classify into six large groups, the 6Ns, considering: nanometer, nanoscience, nanotechnology, nanovisualization, nanomanipulation and nanomanufacturing. However, initially focusing only on $\mathrm{N} \& \mathrm{~N}$ nanoscience and nanotechnology, which contemplates the theoretical academic purist, mathematician, biologist, physicist, versus practical, engineering and commercial, can already be considered two large groups. However, we can refine this apparent classification. As an example, through simple questions, we can note a first classification, contemplating:

- What is the academics concept, idea or product?

- What do you contemplate in nanotechnology in your concept idea, product or service?

- What is the percentage of nanotechnology in your product?

- What is the level of complexity of nanotechnology?

- Following the presented Figure 1, or by referring to the diagram that this paper suggests, for fast classification of complexity for nanotechnology, where do you find your concepts, idea, product or service?

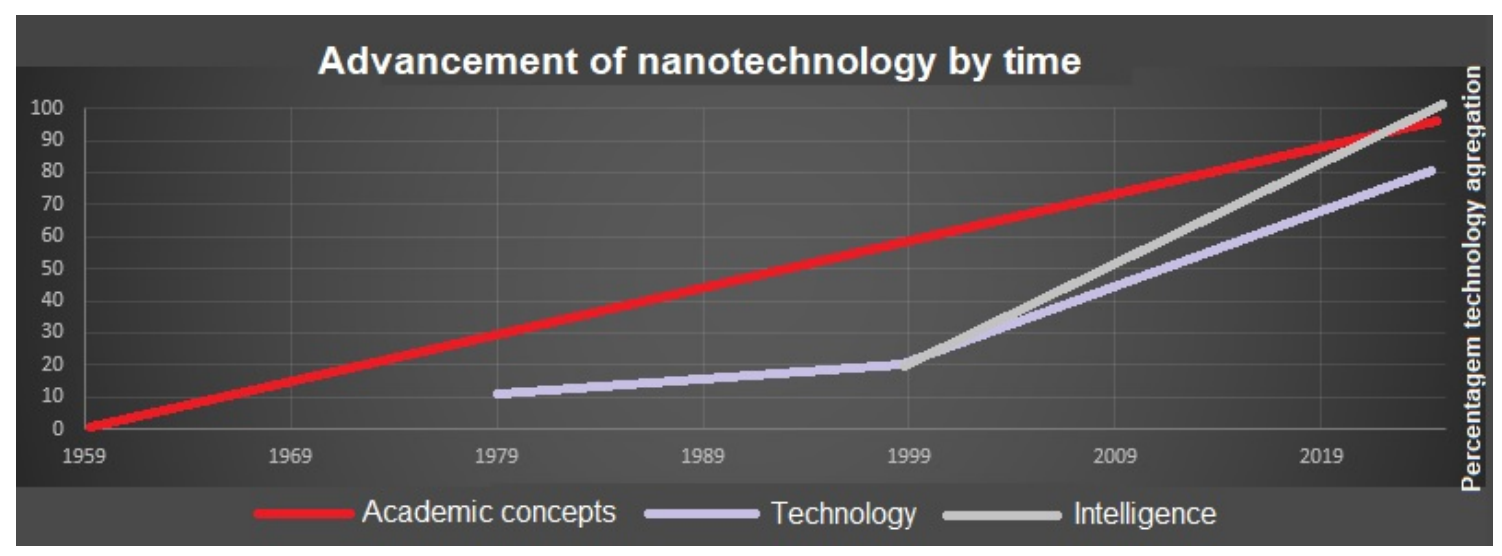

Figure 1. Exponential demonstration of the growth of concepts, technology and intelligence in nanotechnology. Ideally used by means of questions, to reach the level and classification that is the nanotechnology researched. 
Exactly this term, "targeting" fits this proposal well. As purpose of this paper to separate nanotechnology by niches or segmented specialized fields, but in a scientific, analytical and technical way to reaches a rating, for future normalization as well as for standards and specifications. Note that this is not an individual work, but involves modern techniques of crowdsourcing and collaboration of different segments. So, this paper will present specific distinctions to classify, or better order, nanoscience and nanotechnology. The intention is not to purely segment, but only to classify, because the act of sectorizing is depriving the freedom of a science or business. In short, the intention is exactly this, to list and to order in a concise and customized way. Because nanoscience and nanotechnology is something gigantic in information, and as a way of ordering, and making more visible this gigantic technoscience. This is the proposal for greater progress. Apparently, this paper may seem trivial, but its intention is profound for those who understand this branch knows the gigantic importance of what we are proposing in the studies presented in this paper. As example for nanotechnology, achieving something like the engineering standards governing the oil and gas industry is a model to be followed. So, is important include a way to handle immense amounts of analytical data [5]. Separating what is nanotechnology from what is pure marketing in fact is something important, for several factors. The importance of marketing to nanoscience and nanotechnology is vital and unquestionable. There is no problem in using the nano name in trades, products and service providers that have nothing of this technoscience. This rating is even important for scientists, researches or investors to drive more effective efforts. To direct its development without deviations, with serious and concrete actions. As well as for a greater general progress of this science to the generation of its good results with the final technology. Note that this paper taking the point of view of mechanical engineering, in special taking as example success, as the standardizations of mechanical industry, as for example the great work of MSS [11].

The idea of starting this study came after a criticism, a bit nasty, of a friend and student of the subject nanotechnology. To which, when we talked about the nanotechnology market, especially in Latin America, he said that the nanotechnology products are only in cosmetics. Nothing more than aggregates, and formulations containing antiseptic nanoparticles, or mixtures with particulates, blended into some common and simple formulation. Nothing so complex. But I replied that it is not so, especially in a branch that is well developed. Although it cannot be considered as the apex of nanotechnology, we still should recognize that any initiative that can use something of nanotechnology to develop this industry is welcome. If each sector does its part, honestly and with quality, in a short time we will have a great economy based on very advanced nanotechnology. Mature enough to progress in bigger steps, more professional and with more technology. And above all, contributing to the world, in the development of this science. The use of the name nanotechnology without any commitment to science, just making use of its name as a brand, or means of promoting something, if it does not incur something illegal, has nothing much. However, as already mentioned, a technical classification tends to bring professionalism to a subject. And in the case of nanoscience and nanotechnology, means of professionalizing it is fundamental for its effective progress.

\section{A Model for Achieving Nanotechnology}

Any company can use the term "nano" in their products and services, no problem. Before this pre-post we can analyze a case, which although simple, evidences the potential of this technoscience. As an actual hypothetical case: In the early 2000s, nanotechnology took a high degree of popularity in the USA, and of course many people wanted to enter this new futuristic branch. An entrepreneur, even without any notion of its high complexity, ventured into this way, so a case occurred as follows:

"The case of the small coffee shop in NY tells the hypothetical story of an unemployed man, and to reverse the situation opened a coffee shop after getting a commercial location. This one was so small, really tiny to fit a maximum of five people at the front street. The beginning as every entrepreneur has proven, was of fear, uncertainties, and bills to pay without end. Because of the size of the commercial hall being so small, he christened his coffee shop with the name NANO. Obviously, there was nothing nanotechnology in a business like this. But over time, the people who frequented the place began to talk about nanotechnology, and the owner began to read on the subject without stopping. After a long time, it managed to capitalize in a minimal but satisfactory way. And with the professionalization of your business the entrepreneur began to think about growing, and even with new ideas. Over the years, as he had read much on the subject nanosciences and nanotechnology, he had the idea of creating a machine to produce pure coffee, with pressure filter. Thus, he adapted HPLC liquid chromatography-type high pressure liquid chromatography equipment to separate just the caffeine he was interested in. In addition to having created more accurate filters and filters that added flavor to the coffee. After a while, with a friend developed these projects, and patented them. And so, he went from an unemployed to a merchant, and today he is an inventor and industrialist employing several people. With plans of expansion without limits and export to several countries ".

As we noted, in this hypothetical story, but very similar to many cases, is what we defend here! Commercial freedom and entrepreneurship, as well as the free use of the name NANO, nanotechnology in business. Obviously, there are some caveats of insignificant and even ridiculous uses, but the worst and inadmissible restraint is regulatory, is the way 
to the destruction of this huge industry. Dangerous things already exist, such as the synthesis of TTX which is Tetrodotoxin, a potent neurotoxin. Or even the dangerous nerve agent, or rather the long-lived VX gas in the environment, its synthesis of production is even simple in the face of the complexity involved in some more advanced nanotechnology. And yet there are no people producing these dangerous and deadly toxins in every corner. Some $99.5 \%$ or more of people want and look at nanotechnology with an economic bias, so one should not be alarmed. So soon, freedom and doing is what will boost and much the development of nanotechnology.

\section{A First Practical Classification Model for Nanotechnology}

The classification consists of levels, considering the scientific and technological complexity of each item based on nanotechnology. With this classification, it is easier to visualize, define and classify products containing nanotechnology, demonstrating, and making clear to researchers, scientists, investors, entrepreneurs. And thus, indicating better the space to grow and develop of this science. That is, a professional ranking in this technoscience serves to separate what, has nanotechnology aggregated or is the very nanotechnology, of what is just marketing.

The idea, as already said was based upon hearing a comment, perhaps naughty, saying that only cosmetic or some pharmaceutical companies know how to develop products with nanotechnology in $1^{\text {st }}$ generation. Soon I thought the opposite, for being good news, at least there is a sector involved in the whole chain, from end to end, of the concept to sale, in a complete way. But, furthermore, I thought from this comment that a clearer reference to the sector was lacking, that is, as some technical and scientific diagram, as presented in references [2]. Not a list, but sort of a quick lookup chart. As in game screens, in which there are already reached levels of progress. In the future, it will be interesting the possible development of a programming to accommodate the huge listing of data and information on each new production procedure in nanoscience and nanotechnology. These are initial steps towards a professionalism in the technical classification of this science, with technical and commercial purposes. In this way, with much consultation in papers and readings on the subject, always looking for a less conceptual model, and more practical in search of key and analytical points, with the key terms for classification.

In this way, an initial model was developed, this could be improved with data collection to guarantee the degrees of difficulty. Classified at levels that confer from level I to VI, and although the cosmetics sector has been allocated at difficulty level I, depending on the data inserted in the algorithm, in development, added to the difficulties involved, the complexity of the product, if involves complex synthesis or just a formulation based on some simple unitary operation. And from hundreds of variables to classify complexity, the level of nanotechnology-based product is allocated to its classification. Following the model presented in figure 2. The issue for this classification is only to encourage complexity, as well as serve as a thermometer, compass, and guidance for those who invest, produce, invent, research and develop nanoscience and nanotechnology. It takes an immense, conceptual, analytical and careful accuracy not to confuse academic with process or products and services. And so, focus the classification by a clear method and differentiate by degrees of complexity what is nanotechnology in degrees of purity.

- In this proposal, in search for a classification, as already informed, it is not enough to separate the academic from the application, as by the acronym $\mathrm{N} \& \mathrm{~N}$. It takes something more precise, practical and complete. A classification model not limited by the analytical check, but including considering some strategies and mathematic logic, including:

\section{Classification:}

- Concepts, technology definition

- Stabilization of molecules

- Functionalization

- Mooring grade / structural / geometric

- Micro / nanofabrication

- Bionanomechanic and biological mimicry
- New particles / molecules / events

- $\quad$ Synthesis: physical-chemical, biological or mechanical

- Terminology and nomenclature

- Characterization, measurement and bionanometrology

- Health, safety and environment

- Material specification

As we can see from this basic list of topics, to classify nanotechnology solely by analytical results is at least somewhat incomplete. From this point of view, we can verify another approach, by means of an average of the aggregation rate of these items in the object of study, as follows: 


\section{Ranking indicator for identifying nanotechnology level}

\author{
Estimated rating \\ (from low to high level) \\ ( 1 to 100 th) \\ $1^{\text {st }}$ to $20^{\text {th }}$ percentile (worst) \\ $21^{\text {st }}$ to $40^{\text {th }}$ percentile \\ $41^{\text {st }}$ to $60^{\text {th }}$ percentile \\ $61^{\text {st }}$ to $80^{\text {th }}$ percentile \\ $81^{\text {st }}$ to $100^{\text {th }}$ percentile (best)
}

Median rate aggregation over nanotechnology

(1 to $100 \%)$

$$
\begin{gathered}
20 \% \text { (or less) } \\
37 \% \\
60 \% \\
76 \% \\
92 \% \text { (or more) }
\end{gathered}
$$

This estimation may include cost and efficiency based on estimated functionalization data selected between 100 cases studies based in statistic way [12]. It was also consulted as references [7].

The overall rank is calculated as the average of complexity, functionalization, synthesis, innovation, originality, material intelligence, a new molecule, nanoparticles with events, actions or new functional technology. All this for rating the complexity functional nanotechnology and not just exactly by laboratory analytics.

\section{The Percentage of Functionalization as a Parameter of Complexity}

The functionalization of nanoparticles is the model parameter that we intend to consider, like initial standardization to a classification in nanotechnology. Technically, when we think of allocating a ranking, or a way of classifying the type of nanotechnology in a product, service or innovation, we have defined that analytical tests is one of the resources to confirm the purity and percentage of the added nanotechnology. Making use of statistical calculations, as per percentiles for reaches a ranking. There are many companies that simply add additives, without any nanotechnology work, as a quick way to position themselves in the nanotechnology market. In fact, there are many companies and researchers who acquire fractionated inputs of nanoparticles, material usually in powdered state, simple particulates, nanometre-sized granulates, e.g. silver nanoparticles that are antiseptic, bactericidal, and proven to be anti-infective in some cases. However, nothing to synthesize the nanoparticles, or synthesis, functionalization, nothing, much less develop a molecule in fact. Much less functionalize the same. And in this way, they add these particles to some liquid forming product, gel, spray or paste. Just as they add this material in capsules or some product, and in this way, they position themselves as nanotechnology companies. Being nothing more than cosmetic products, pharmaceutical low or medium technology aggregate.

There are no problems using these techniques to position yourself in the market, it is even good and recommendable. If there are physical-chemical tests and good manufacturing practices, as well as not affecting customers or the environment. And being a product accepted by customers, there is no problem. The question we raise is that there should be a ranking to differentiate nanotechnology, in degrees of complexity. It would not be fair for a company that only uses the name nanotechnology to be matched with another company that spent 20 years developing a molecule that is functionalized, or stuffed with some known asset. At this point, borrowing some pharmaceutical terms to define well a subject so complicated to explain simply. Thinking this way, for those who are in the field, you know that nanotechnology really needs a ranking, something that allocates a degree of "purity", or a product or service that contains nanotechnology in products or services that are just marketing. However, even as a qualification and a differentiation, it is important to have a score and a differentiation, even for academic matters. And so, serve as a compass, even for investors. Nevertheless, statistically classifying nanotechnology by functionalization index, and the complexity of this functionalization is already an advance in this way. We can think of other approaches as well [9].

\section{Sort by Process versus by Dimensional}

A commercial classification. A classification for the level of technology in products with nanotechnology versus the degree of purity or existence, or not, of containing added nanotechnology. In this paragraph, we present a classification procedure for products based on nanotechnology. To separate products by their level of aggregate technology. There are many products that tend to be more marketing than real technology. Yes, really, any help to increase the development of nanotechnology is important. However, it is also important to separate what in fact has high level and low-level technology. This is a first draft of a procedure, a first model in development of classification. It is a great reference for nanotech developers. This classification is important as an indicator for future progress in the area. In addition to serving as a tool for promotional agents, scientists, developers, interested industrialists, investors and researchers in nanoscience and nanotechnologies. These benchmarks will serve as a 
procedure. It should allow future identification in the route of a future map of innovation, research, classification, and so on. In this way, a reference list for products and services for classification is presented here. In this example, there are some exemplary products, but other innovations products can be added, adjusted or replaced in these positions. It is important to consider the complexity of this work, and not to confuse this proposal of this paragraph, presenting an express classification of products and services with the most complex proposal of analytical gauging, proportionality and disproportionality in nanoscience and nanotechnology. Faced with the proposal of an eminent need to have standardization through standards for nanotechnology. To facilitate access to data and analytical information of large volumes of information in a practical way. As we see in standards such as ASME American Society for Mechanical Engineering, and ASTM American Society for testing and materials. Engineers around the world, when making a new product, project or developing a new technology, do not have to resort to analytical laboratories for complex and expensive tests. To simply characterize a material, or even to select a specific material that best fits a specific use, meeting the required requirements. And what we propose in this article is exactly to take these cases of engineering for a necessary technical standardization of nanoscience, through diagrams, graphs and tables, presenting the characterizations and specifications of most common uses of materials, processes, dimensional and analytical data. Including this, even for commercial use classification. However, as this work is immense, it is necessary to open a working group to this end, through networking for crowdsourcing or even peer production. Involving several people and academies generating this standardization to nanoscience.

As shown in figure 2, below, an express and commercial classification model is presented to identify the positioning of nanotechnology under study. No matter if it will be product, service, process, aggregate of technologies. After passing through several filters, the item analysed receives a score, and is then positioned in this listing, which positions the object of study in a level of technological complexity. Level I considered easy to level VI considered excellence, and difficult to replicate, because it contains high level of aggregate nanotechnology, originality and innovation. This proposed model index spectrum will serve as a compass to guide investors, researchers, students and even the press. Companies can also earn value as their products are better positioned in this ranking.

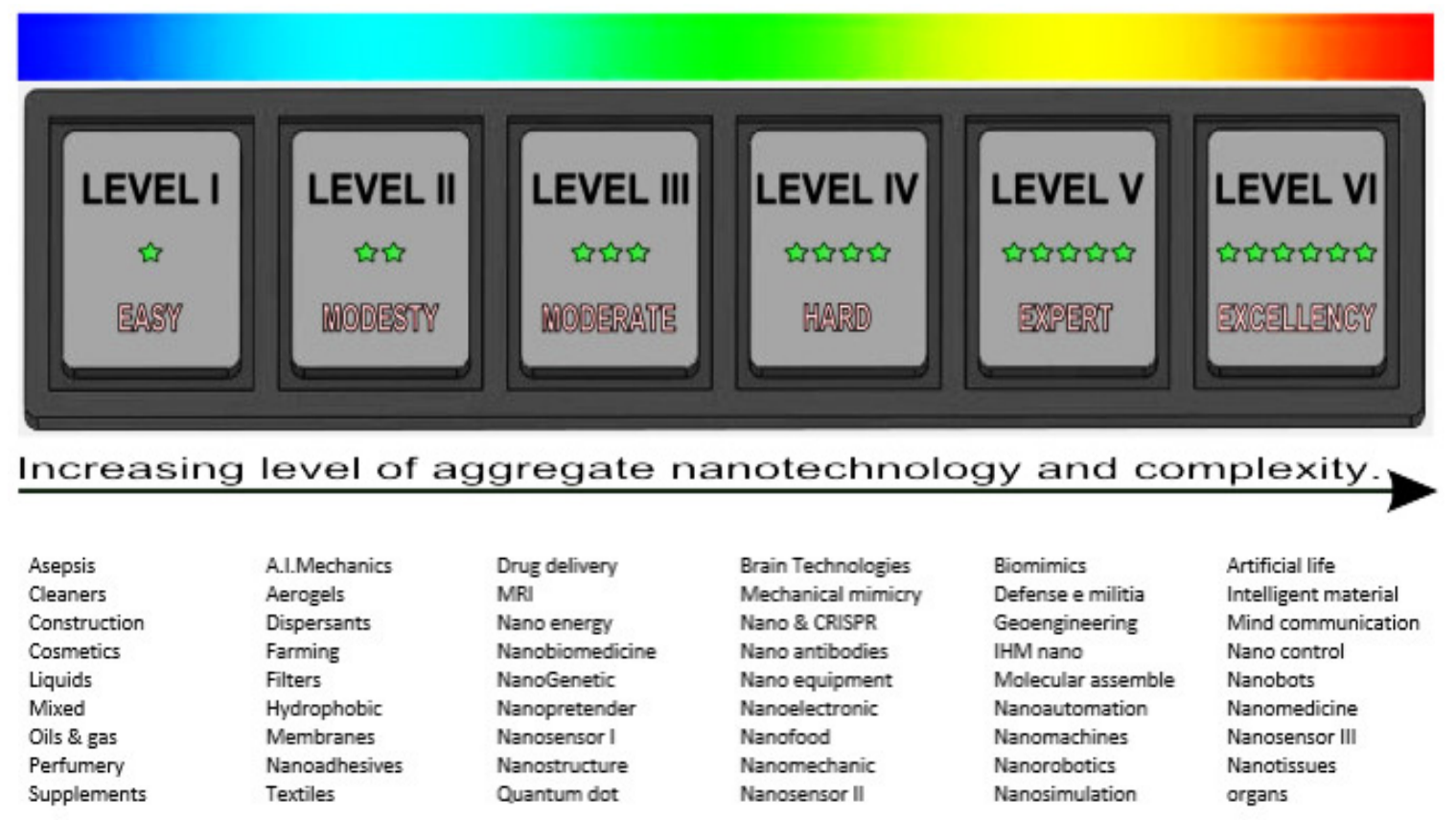

Note: This basic spectrum classification is a template for placing each product by its level of technology. The less stars the product tends to have less technology, being a product of only mixtures, use of nanoparticles dispersed in fluids or just marketing. It is important to note that services, characterization and analysis in general do not fit this classification, are presented later. To reach this model was consulted the techniques demonstrated in the literature [4].

Figure 2. Description of the level of complexity, in nanotechnology products, process or studies. 


\section{Description by Level}

- Level 1 - It is the nanotechnology-based product that earns 1 star, considered as an easy aggregate technology.

- Level 2 - It is the nanotechnology-based product that earns 2 stars, considered as modest aggregate technology.

- Level 3 - It is the nanotechnology-based product that earns 3 stars, considered as a moderate aggregate technology.

- Level 4 - It is the nanotechnology-based product that earns 4 stars, considered as advanced aggregate technology.

- Level 5 - It is the nanotechnology-based product that earns 5 stars, regarded as an expert in aggregate technology.

- Level 6 - It is the nanotechnology-based product that earns 6 stars, considered as aggregate technology of excellence.

\section{Organization and Classification of Nanotechnology}

A satisfactory way for classifying and organizing both academic and technical considered dimensionally by I.S. physic International System $10^{-9}$, or by scope $1-100 \mathrm{~nm}$. So, we can organize it for the 6 Ns. To include in these six Ns:

- $1 \mathrm{~N}$ - Nanometer (contemplates dimensional, I.S. classification $10^{-9}$ and scope $1-100 \mathrm{~nm}$ ).

- $2 \mathrm{~N}$ - Nanoscience (contemplates academic knowledge and information organization).

- $\quad 3 \mathrm{~N}$ - Nanotechnology (contemplates the technology fruit of the practical application).

- $\quad 4 \mathrm{~N}$ - Nanovisualization (contemplates visualization techniques in the nanometric world).

- $\quad 5 \mathrm{~N}$ - Nanomanipulation (contemplates manipulation techniques in the nanometric world).

- $6 \mathrm{~N}$ - Nanomanufacture (contemplates the techniques and processes of manufacture to obtain the nanotechnology).

\section{The Importance of 6Ns in the Organization and Classification}

It is not about contextualizing the classification between 1 to $100 \mathrm{~nm}$, or to separate by academic and practical as $\mathrm{N} \& \mathrm{~N}$, but to add in this simple definition something more precise and comprehensive. We have that the nanometer refers to the dimensional, by the I.S international system of physics represents $10^{-9}$, and as scope of performance, by ASTM 1-100 $\mathrm{nm}$. As we all know, nanotechnology involves a science of dimensional boundaries. That is, clearly this technoscience is conceptualized in a dimensional scope. Technically in the range of 1 to $100 \mathrm{~nm}$, however, products and services with aggregate nanotechnology can work in combination. With components in meso-scale, micro-scale, nano-scale, and lower scales. All in one product, service or study. In this context, the classification will need passes through a funnel, as in the case of the analytical diagrams proposed in this paper, in which the bottleneck defines its classification. May be by complexity, refinement, background technology, functionality, new innovative technologies, to pass by the filter, like new molecule, innovation, nanotechnologic based and so on. The analysis done here is that this classification or definition is more global and for specific products. Not being complete as a definition for nanotechnology, going from the global specification to a more precise specification. A more accurate classification of purity involves a more analytical classification, something like the degree of functionalization, or events contained in a particle, something between 1 and $100 \%$ functionalization. A classification, e.g. from 1 to $100 \%$ as score, how much in a simple wall nanotube was functionalized, and what level of complexity of this functionalization? With such a classification, it is possible to obtain a more precise, technical definition, a truly precise specification of the level of nanotechnology aggregated in some product, service or study.

\section{Considering Synthesis and Process as a Means of Classification}

There is in fact a difference in complexity between syntheses. The physic-chemical synthesis, that of finding affinities between atoms and molecules, and connecting them by diverse processes, something like putting together or assembly atom by atom, an original process of bottom-up, in spite of being complex. And this process is a form of classification for nanotechnology. However, there is a great difference between this process and the one of mechanical synthesis, in degrees of more complexity. In fact, it could be said that mechanical synthesis would in the near future work with physic-chemical synthesis, and thus complement one another in some way, in nanomanufacturing processes. However, in theory, the level of a mechanical synthesis involves the manufacture by atoms, mechanically and highly precise, by mechanical biological processes to perform the work of physiochemistry. Mechanical synthesis is more complex, involving micro / nanofabrication and a sequence of coordinated operations in an absurd logic of operations. However, after this production domain, there are no limits to what can be produced with mechanical synthesis operations, ranging from the production of molecules, even to robots. And above all, the biological mimicry cells considering its interior, contemplating DNA / RNA, artificial life attempt, and even futuristic applications. With the progress of bioengineering, include techniques such as CRISPR, and all new intelligent nanoparticle techniques. With intelligent charge for individual treatment per individual database, in addition to individual treatments directly in individual cells and tissues. The point is that mechanical synthesis can be rapid and uniquely automate the process of assembling atoms, whether automating physicochemical synthesis or other processes. 


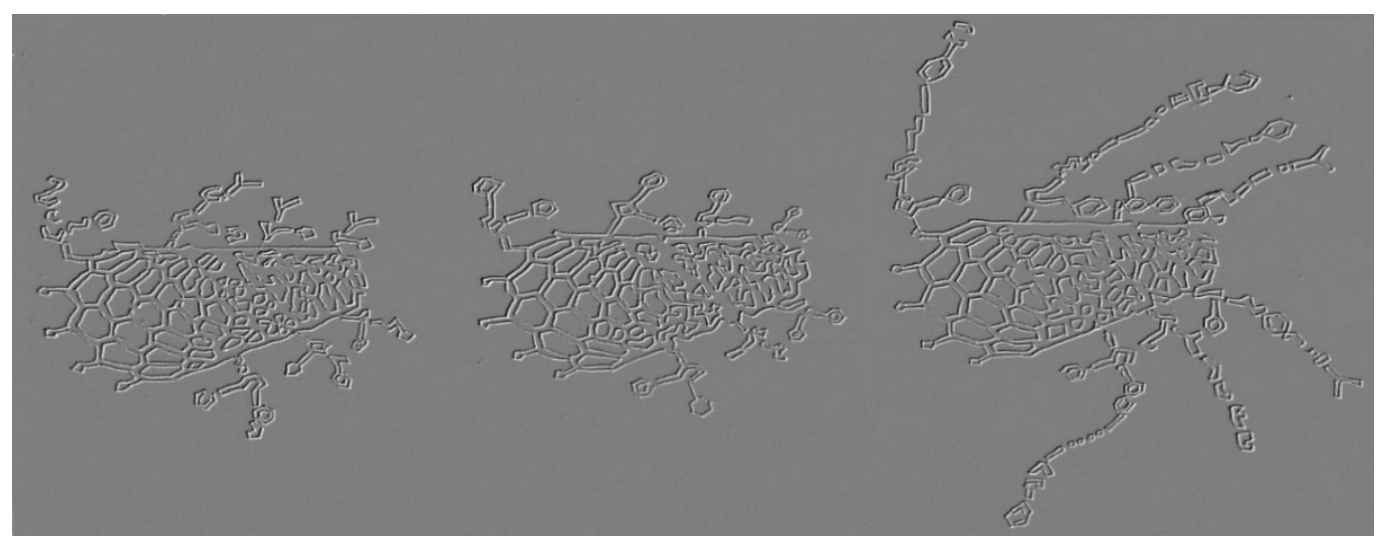

Figure 3. Images by nanoscope visualization for functionalization of nanotubes. Nanotubes functionalized in $5 \%, 10 \%$ and $15 \%$ organic chains in their surface area. The higher the percentage of functionalization, the higher the score to reach the level for technological classification. However, if in addition to functionalization, there are other original technologies or innovation, the higher will be the score.

"There are syntheses and syntheses, while some involves a simple blend, others may involve hundreds of biological and combined processes, micro / nanofabrication and unprecedented industrial complexity."

In addition to having other means, the degree of functionalization is a good indicator. For example, as in Figure 3, we see an example of a nanotube being functionalized, with $5 \%, 10 \%$ and finally with $15 \%$ functionalization in their surface area. While only measuring functionalization is not enough, it is a high complement to a future and due ranking for nanotechnology.

\section{Methodology}

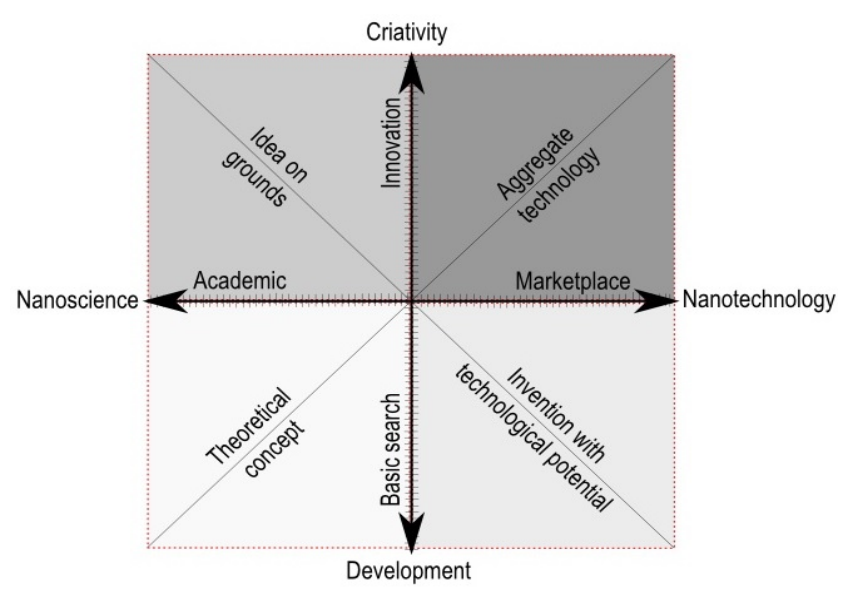

Figure 4. Map for description and identification the actual position of nanotechnological analyses studded.
Spectrum technological density by level in nanoscience and nanotechnology is a system to classify different positions of ranking levels of advancement in the technical-scientific density on one or more geometric axes. To symbolize independent dimensions and levels reached or leased of the treated technology. Within the concept of existence of a concentration of science and technology in a product, service or concept of nanoscience or nanotechnology. A fast and express way to filter it for organization and classification. Details can be seen in figure 4 by a map description for locate the object of study.

Making use of mathematics and logic is a fundamental item for the classification, organization, coordination and taxonomy to nanotechnology. And to include in this classification is presented a table with the basics of logical mathematics for an effective categorization of nanotechnology that is just beginning work, and will involve all the research centers in the area to complete this work. As we noted in table 1, we have the basics of mathematical logic for this purpose. To use math and logic to help create techniques to filtered as said; the organization, coordination and classification for nanotechnology.

The steps to a mathematical logic in the organization, classification and coordination for nanotechnology, for future standardization is something complex and not limited to analytical. It also involves engineering details and product generation. To include steps such as amplification, immersion, prototyping and realignment. Because, for a definitive standardization in nanotechnology, it is necessary to consider all the details involved, including the commercial question in some cases, and not just pure analytics. 
Table 1. Using mathematical logic to categorize nanotechnology into classification criteria.

\begin{tabular}{|c|c|c|c|c|c|}
\hline Logic & Definition & Score & Ranking & Formula & $\begin{array}{c}\text { Index } \\
\text { Nano }\end{array}$ \\
\hline Classification & Group objects by similarities. & $\mathrm{A}$ & Value & $\mathrm{A} *$ Value & Result \\
\hline Comparison & Logical process of comparing sets. & $\mathrm{B}$ & Value & $\mathrm{B} *$ Value & Result \\
\hline Conservation & Quantity does not depend on form, order, organization and position. & $\mathrm{C}$ & Value & $\mathrm{C} *$ Value & Result \\
\hline Correspondence & Is to attach to each item a value, an assignment. & $\mathrm{D}$ & Value & $\mathrm{D} *$ Value & Result \\
\hline Inclusion & Operation to cover one set by another. & $\mathrm{E}$ & Value & $\mathrm{E} *$ Value & Result \\
\hline Sequence & $\begin{array}{c}\text { Defined by the sequence elements that are done in a regular and linear way, } \\
\text { always maintaining the same relation with the corresponding ones, forming a } \\
\text { pattern. }\end{array}$ & $\mathrm{F}$ & Value & $\mathrm{F} *$ Value & Result \\
\hline Seriation & Arrangements together of objects, to maintain with their correspondents a \\
relation of difference. & $\mathrm{G}$ & Value & $\mathrm{G} *$ Value & Result \\
\hline
\end{tabular}

This listing presents the basics of math for classification, organization and coordination of data and information. For a mathematical search that can represent and answer the question: how to write mathematically, to represent nanotechnology by its degrees from more lows to the highest level.

What questions are needed to define the degree of complexity? What would a scientific, engineering diagrams, abacus, tables, statistical analyses references look like to technically classify nanotechnology. To start building these references it is necessary first to generate the necessary key questions by making use of logic, making use of logical math, as shown in the table. An important item to categorize nanotechnology is to start with the right questions, and with a standardization of these questions, we can arrive at an initial classification standard. Certain global technical questions can serve as a funnel to start a taxonomy in nanotechnology. With this, we will have the initial steps for the construction of flowcharts, diagrams, and consequently diagrams such as Mollier's thermodynamics and various scientific, engineering diagrams or abacuses references for nanotechnology. With this apparatus, we will have at hand a broad set of tools to categorize and classify nanotechnology effectively. The best way to arrive at standardized analytical data for nanoscience is mathematics, especially empirical and analytical statistics. By the statistics there are the subsidies to organize the data of the analysis and collected data results from experiments of characterization in nanoscience. With the organization and ordering of analytical data results, innovative high precision diagrams can be made. Resulting in linear models and decision trees, as well as in combined graphs and highly scientific models, as we can see in the treatment of data in software such as Mathlab $^{\mathrm{TM}}$. Analyses in HPLC and its representation are examples of this possibility, as we can see in the reference: "The dendrogram shows the merging of samples into clusters at various stages of the analysis and the similarities at which the clusters merge, with the clustering displayed hierarchically" [13]. By this means methods and standards can be achieved for nanoscience, through samplings or even direct analytical tests. With the advantage of the current increased computational capacity. It is possible to further refine the data and information, which after being organized can become scientific diagrams, tables and valuable references for nanoscience. Some questions to start this long process of work could be, when someone presenting an idea, conception, product, service or process with nanotechnology, this be submitted the following as initial questions in a logical way:

1. Is it a new idea or innovation?

2. Does this technology already exist?

3. Is there a process or analytical tests?

4. Is there any prototype, test or virtual model?

5. Already have an academic concept?

6. How many hours or years of analytical study?

Then, after going through the initial filter questions, continuing for narrowing and refining it passes through the classification funnel of a diagram, as follows in figure 5, as a simple model proposed to be ordering the necessary basic information's about the study, product, process or service nanobased.

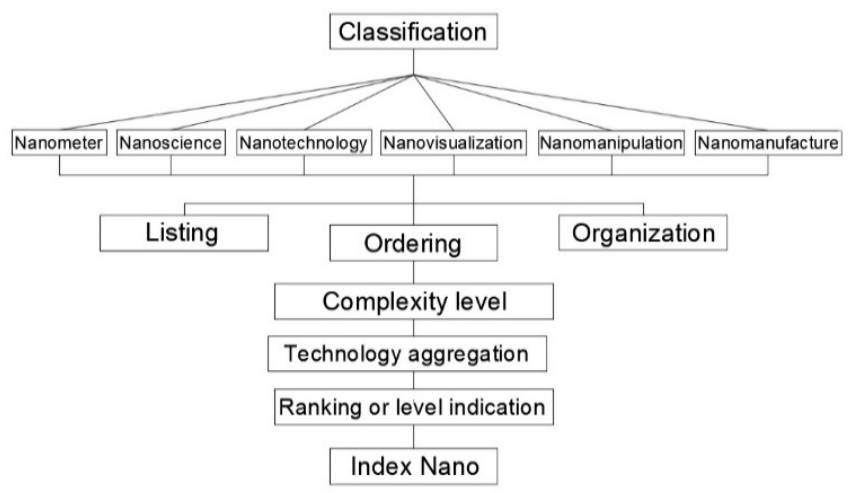

Figure 5. To organize first the necessary information's, and define the type of nanotechnology. So, to organize the type of nanotechnology, as well as to verify if the same product, service or process presented has in fact aggregated nanotechnology. And finally, which its score or level of technology reached to finalize with an index.

And after the study, idea, design, product, service or process with nanotechnology go through the set of logical questions, through this flowchart, and some other analytical filter. It must be submitted to a logical flow chart, as we will see next. To effectively prove whether the analyzed items contains added nanotechnology and the level.

\section{An Initial Flowchart for Fast Sorting}

When we want to organize and classify some science, information, as a taxonomy to nanotechnology. It needs 
strategic means that go beyond pure statistics. So, nothing is better than an ordered listing of the variables involved, with their proper classification and their coordination and organization, by means of a flow chart. Taking as a model the discipline and science biology, with the taxonomy, as used for classification of living beings. The idea presented in this paper is to use this model for the proper classification of nanoscience and nanotechnology. Following this principle, we present below an initial flowchart to attempt to quickly classify the level at which the research, product or use of the term nanotechnology fits. This flow chart is complementary to the classification diagrams and other references for $\mathrm{N} \& \mathrm{~N}$ which is the purpose of this paper. With this alignment, we can begin to define a logical flowchart to filter the nanotechnology object under study. Analytical data and especially scientific diagrams deserve special focus. In the case of this flowchart presented, it intends to act as a filter, a complement for the future scientific diagrams, tables, graphs and other references that can be consulted individually or together to obtain data and information in nanoscience and nanotechnology works. Nevertheless, in figure 6, we can visualize the next step proposed for narrowing and filtration more the information for an effective organization, coordination and classification for a ranking in nanotechnology.

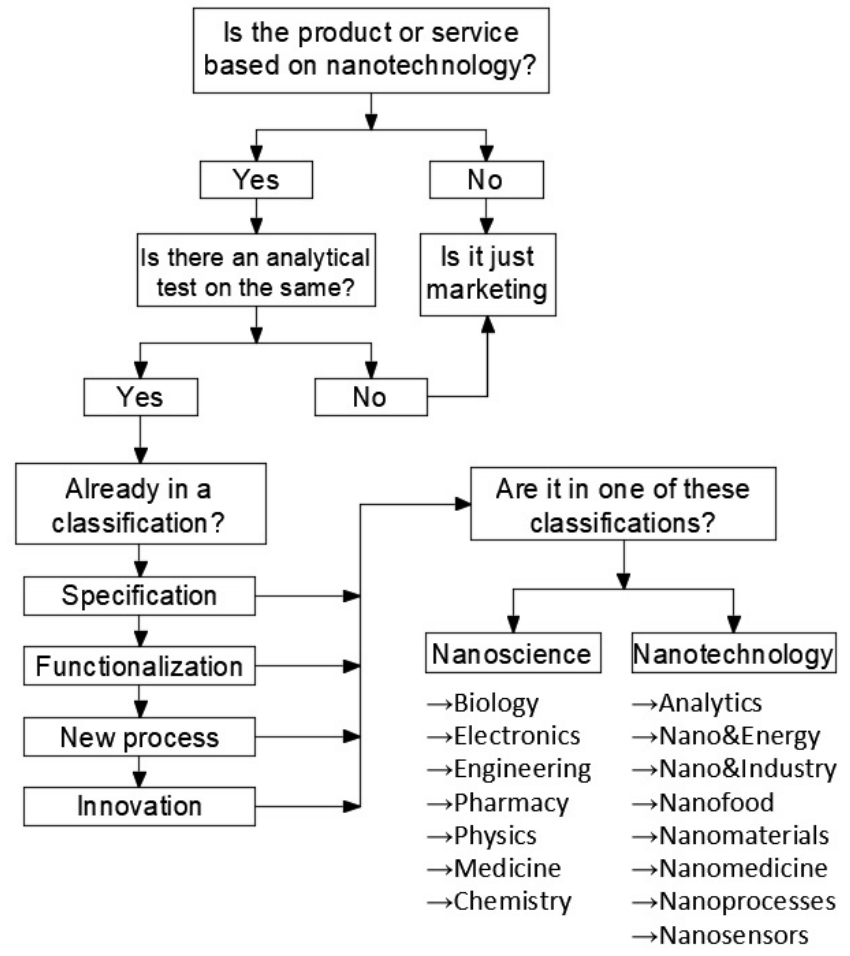

Figure 6. Express verification scheme to identify where the use of the term nano is located, whether for products, processes, research, service or business. A new and effective step for narrowing and filtration the item of study to organize and classification
Then, if the study, analyses, idea, design, product, service or process with nanotechnology have gone through this classification, these filters and funnels, effectively proves that there is nanotechnology aggregated. Finally, the same receives a score, on scientific technical scale, and a classification of level of complexity. Through this ranking investor, scientists and the public can effectively understand the level of aggregate technology being treated, researched or even consumed. And from these studies there may be analytics references, as graphs and diagrams with high scientific capacity of effective and commercial use in practical engineering nanotechnological.

\section{Scientific Engineering Diagrams for Nanotechnology}

The need to build a scientific engineering references diagrams is something extremely necessary. The next step to organize and classification nanotechnology follow this idea. Scientific engineering diagrams are extremely practical and functional, and will be the mainstay of practical nanotechnology. Classification of the level of nanotechnology by means of an engineering diagrams. It is a fast step to develop N\&N nanoscience and nanotechnology. Any engineer or people don't need every time, in your study, development of product or idea based in nanotechnology, repeat the same analytic procedures, all time, so expensive, and requiring a long time. Take as success example, the actual way of work with mechanical engineer. In special for refineries, onshore and offshore, for piping, when use tables, diagrams and calculus standardized as e.g. ASME and ASTM, etc. It's an effective and fast way to get analytical results of complex mathematic and laboratories. In this way, the performance of the engineering work triples, and it becomes possible over time to innovate and think about improvements and new products and even innovation with the time gained. And this classification following the proper concepts of classification mathematics, and taking as a model the process of taxonomy of biology, in conjunction with scientific diagrams. So, we can get a ranking / score of nanotechnology level, for a future index for Nano. An engineering standardization for nanotechnology. A fast way to streamline work and classify new materials, products, services and processes. The following is an example or future model of an abacus in figure 7 to define and classify the type of nanotechnology. In relation to the classical scientific branch of the nanotechnology in question. And define by the analytical diagram the level of this technology. 


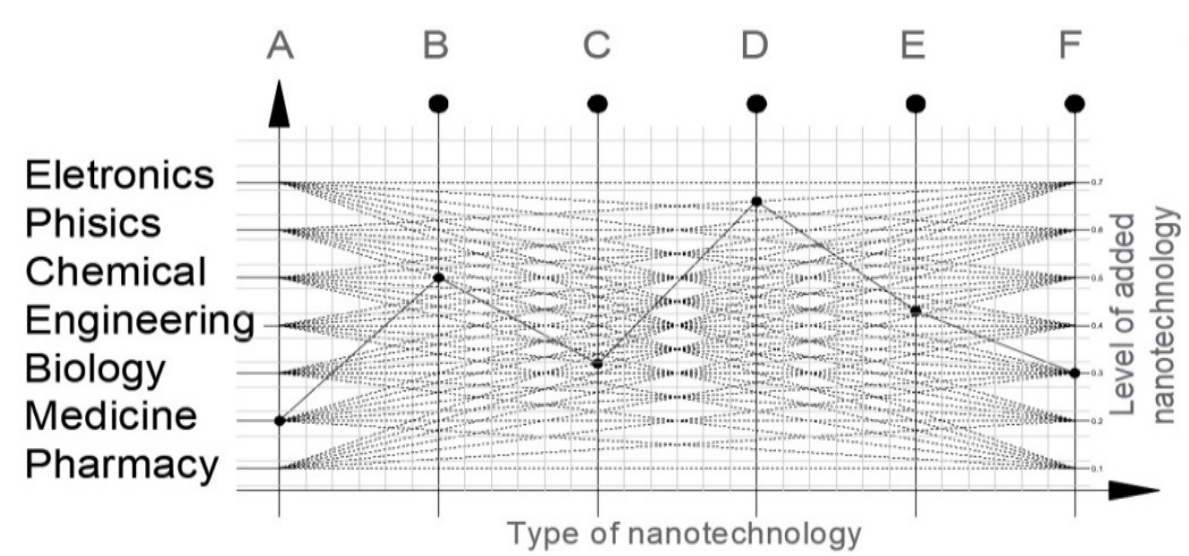

Figure 7. Classification of the level of nanotechnology by means of an engineering scientific diagram/abacus. As described in this classification diagram for nanotechnology, the letters in the axes represent: A: elementary, B: basic, C: multiple, D: structured, E: constructed, F: intelligent. The main line represents the level of technology of analyzed product. This is an example for express classification possible. After scoring each item with the type of nanoscience or nanotechnology under study, we obtain a score that can be consulted in another diagram, and thus obtain a ranking or score of the technological level embedded. Recalling that thermodynamic diagrams are examples and references in their importance to nanoscience and nanotechnology. With large volume of variables and abstract and scattered calculations that need to be ordered.
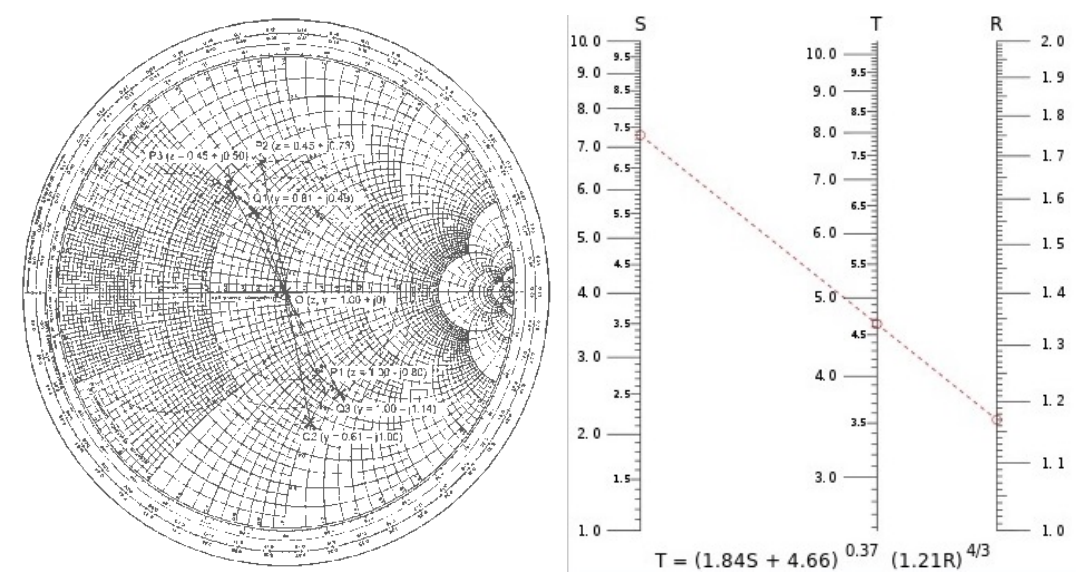

Figure 8. The first the Smith diagram and then a calculation diagram with several variables. This is a model for future diagrams for nanotechnology. Success models to serve as examples for future scientific and engineering diagrams for nanotechnology. For analytical checking high density of abstract data and information. Thermodynamics is a great model for nanoscience. Having a lot to teach for nanoscience.

The importance of using analytical diagrams, abacuses in tables engineering is proof of the immense potential in these resources for nanotechnology. Here is an example of what this paper is about. In figure 8 we have the Smith diagrams / abacus and then a diagram for obtaining results with several variables in an abstract system. The need to organize and treat excess abstract data for nanotechnology does not escape much of what thermodynamics should teach us. As in thermodynamics, there is a conjuncture of dispersed variables, based on characterization and analytical tests on an immense data cloud. As we can see, diagrams are simplifications and shortcuts for obtaining complex results from data, information and arduous analytical work. It is a way of automating the immensity of analytical data in nanoscience. In cases that requires more attention through computational dashboards, or simply graphs, tables and scientific analytical diagrams.

As demonstrated by the examples, science, engineering, and technology progressed rapidly after the creation of simplified diagrams, tables, and complex data organization.
The organization of scattered and abstract data. When there is an excess of data and abstract information, especially analytic information, there is nothing better than to allocate this mountain of information in an orderly way. Making use of various means to sort scientific, analytical data such as functions and mathematical calculations to deal with excess abstract data. And as close to practical uses, in the form of graphs, diagrams and consequently even in software that can work together with practical work. As we can see in Mollier's diagrams for thermodynamics today, abstract data query presents results in a matter of seconds, through queries, diagrams and abacuses in dashboards or software's. This paper points out the need for mathematization of nanotechnology, to simplify its simplified characterization, pointing to diagrams such as those of Richard Mollier, as in figure 9. Diagrams like these, but specific to each analytical study of nanoscience. Engineering diagrams like these will save time and help progress with nanotechnology. Only with an organization at this level can we have a satisfactory classification for nanotechnology. 


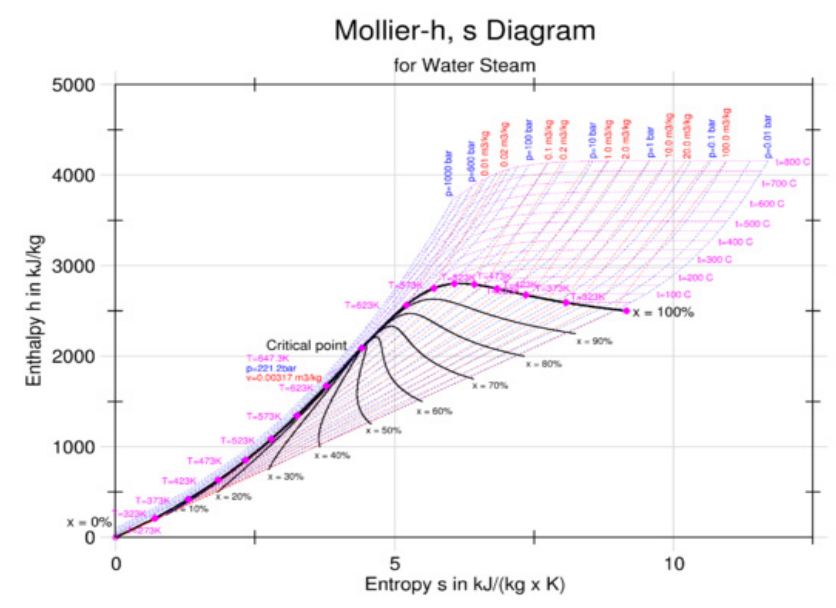

Figure 9. Mollier diagram - For studies in thermodynamics. An elegant way to present accurate, or very close, results of complete calculations obtained by many unknowns and solved abstract data.

The question of this type of work in such a wide area as nanoscience involves time, cost and much collaboration, for each specialized sector to carry out its specialization diagram. This type of traditional diagram requires arduous academic and even laboratory work to be accomplished, finalized with complex mathematical functions and calculations. This proves the importance of following these steps for nanotechnology, converting results, concepts, characterization, and analytics into diagrams, abacuses, graphs, tables and quick-query maps that will result in unprecedented expertise in nanoscience.

\section{The Price of Simplicity Has the Cost of High Complexity}

Mechanical engineering must teach nanoscience to its practicality, practical agility and speed. Not limited to a purely academic purist discipline, but seeking practical results. Obviously, the ideal is a marriage between academic purism and engineering practice. And that exists through scientific diagrams and standards, based on thousands of analytical and characterization tests. It's not a one-man job. There are cases of building scientific and engineering references, tables and diagrams that have taken years with a multidisciplinary team, each one performing specific calculations and analytical graphs. And after a lot of work, all the graphics of this team when being put together in order we have a scientific diagram for simplified consultation. The proposal of building consulting diagrams for practical nanotechnology is an arduous, long-term task, but once completed, we will have something. As we can see in thermodynamics, with ammonia graphs, with a specific volume of gases, or diagrams of Mollier, who are great examples, or even in mechanical engineering, with various piping and valves diagrams, tables and calculations.

And in the case of nanoscience and nanotechnology, organizing so much information is something that requires diagrams for agility. In short, while the academic field and laboratories by generating diagrams and analytical references, will be contributing immensely to the full development of 6Ns. Improvement is an arduous task, which results in information density, but with intense simplification, there will be speed. In the case of nanoscience, in statistic math, calculus for diagrams to specify or select the specific volume of materials, as example, in differentiated densities or mechanical resistance with addition of nanotubes versus graphene versus Buckyballs. Or in the case of nanobioscience, bioscience in general, e.g. a table contemplating all biological possibilities of active fragments for antibody work. Or a scientific diagram to show analytical results of different biomarkers, under different conditions and environments. Various applications in human tissues cases. There are a multitude of specialization groups that can collaborate effectively in their field of expertise.

By means of diagrams, after analytical tests, they could present the mechanical, thermal and electrical conditions under different conditions. And with this information it would not always be necessary to carry out analytical tests with each use of these materials under practical application conditions. After this extensive work in search of diagrams and tables for nanotechnology, simplification will take shape and analytical work can be used in practice, with great effectiveness and speed. These diagrams to handle high volume abstract data, analytical test results and nanotechnology characterization will represent the evolution of new businesses. Benefiting everyone with simplified, fast and low-cost techniques. Enabling innovation and generation of new products, processes and innovation in general.

\section{Results}

Before the studies during the making of this paper was analyzed a series of theories, concepts, observations and reality, to organize nanotechnology. Organizing but not by its terms, because the terms are indeed unique, and spreading in their own complexity and interconnections with other terms. In this case would be a better work presented in a dictionary, which is not the purpose of this paper. Rather, present an assessment, ranking, and level for nanotechnology. Therefore, was used simplified complexity for the organization of nanotechnology through combined scientific diagrams and tables. And we have as a result, through the studies of this paper, that the complexity that must be the thermometer to define the level to which the nanotechnology in question is inserted. That is, it is not just a classification for products with no functionality, in keeping with high development nanotechnology. As a result, is presented ways to reach a standardization for nanotechnology, by means of scores, rankings and how to bring this subject to the modern means of working together. To include, the possibility of taking this work to group work, crowdsourcing, peer production and networking. Each specialized group works 
for generates scientific graphics, diagrams, tables, etc. While any study and product is important to the progress of nanotechnology, it is important that within the $6 \mathrm{Ns}$ we have a classification of levels as presented. And they may receive an index by ranking of complexity. An example can be seen in figure 10 below.

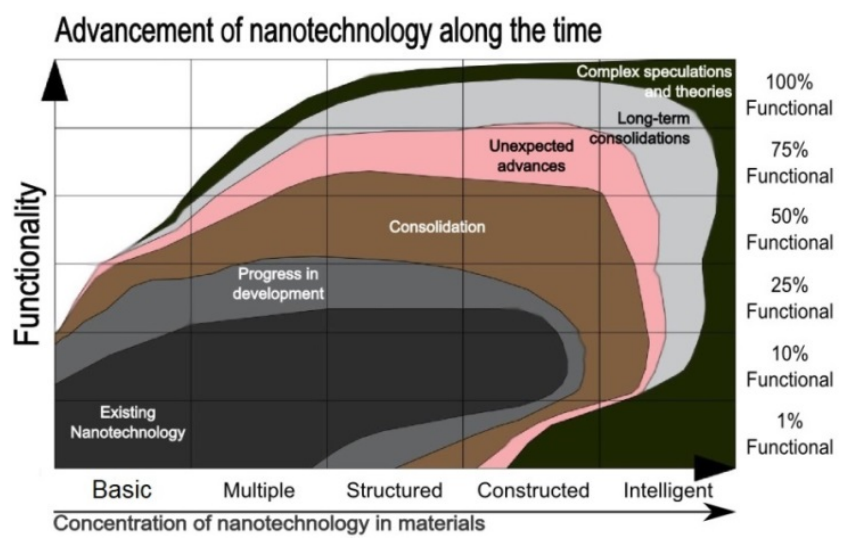

Figure 10. [6]. Representation of the progress of nanotechnology in relation to time, in technical levels by the degree of complexity and of added nanotechnology concentration.

And for this, in addition to the indication of the need for engineering scientific diagrams and abacuses, demonstrated throughout this paper, we have a demonstration of possibility in result. And so, we got a chart that shows the expansion of nanotechnology by time. A chart that summarizes how the progress of nanotechnology will take place over time. Emphasizing that along this advanced technology progress through time, will be given by the addition of functionalization and the dynamics of high control and adding intelligence to materials. All due to the high degree of intelligent interfaces and communication, between different dimensional and complex materials even more differentiated. Something like a nanosensor that captures information in a complex biological environment, transfers into electrical signals that communicate with digital system, translating all information into an assistive system, generating results. But to reach such a high level of advancement in nanotechnology, the need for standardization is evident. A standardization for nanoscience, as we see in thermodynamics or mechanical engineer will accelerate the advancement of $6 \mathrm{Ns}$ like never. This proves the need for engineering diagrams to progress faster and better. With diagrams, it is not necessary to characterize and always carry out analytical tests for each study, product or service performed. The time gained, the quality and the speed in the progress of technologies will present and consequently a clear standardization, e.g. taking ASME for mechanical engineering and too ASTM [14], are cases of success.

\section{Conclusions}

As a conclusion, we can see that for an effective organization, classification and coordination mathematic by data and analytical information, comprises a larger conjuncture. Far beyond purely analytical data. It is not enough to arrive at a classification by pure functionalization as punctuation, but by a conjuncture of technological complexities, and not only by a specific technical analysis. We could directly add a ranking for nanotechnology to include the degree in percentage of functionalization of nanoparticles, however a classification would leave a thousand other considerations vague. One of them is the fact that it is considered that nanotechnology as a technological platform can occur without the particle functionalization. As in semiconductors, as well as in structural reinforcement, and in biological areas of mimicry. That in some cases, are not exactly functionalization, but the very structuring of complex organic materials. Therefore, effectively classifying nanotechnology requires a lengthy procedure of organization, classification, listing techniques, terms and definitions, as well as their combination. That is, for an effective process of fair classification, a better maturation of this technoscience is still necessary. Through organization and classification by scientific diagrams and standards, as indicated throughout this paper, the production of scientific engineering tables, diagrams, abacuses, tables, e.g. the Mollier chart model for gases and thermodynamics. Therefore, as a conclusion to this paper, we arrive at this result, the fact is that understanding this work will understand that it has immense depth, and a huge fast power to the progress of nanotechnology. Still to be achieved, and starting from this route presented throughout this paper. Special groups of study of fragments are important, as in the taxonomy of biology, there are groups of specialized study in each biological fragment, in which when they come together they result in high quality of information. Thus, it is important to note that this work does not alert and calls for the need for the term crowdsourcing, peer production, and the need for continuous collaboration and organization. Especially for a discipline as immense as nanoscience. To reach the level of standards we see in mechanical engineering, with its charts, tables, scientific diagrams for nanotechnology for practical uses, there is still a long way. To which the ranking will be only one step in nanotechnology standardization. And to reach these standards to nanotechnology it is necessary to make use of several methods, including the ANSI/canvass method, peer production, crowdsourcing and all collaboration for a real standardization for nanotechnology.

\section{REFERENCES}

[1] Feynman, Richard, Robert Leighton, and Matthew Sands. The Feynman Lectures on Physics. Addison-Wesley, Reading, Massachusetts: 1965. Vol. III: Quantum Mechanics.

[2] U.S. Department of Commerce - National Bureau of Standards 
- Table and Mollier Chart of the Thermodynamic Properties, C1974.

[3] Eibe Frank and Ian H. Witten - Data Mining, Fourth Edition: Practical Machine Learning Tools and Techniques (Morgan Kaufmann Series in Data Management Systems) - C2016

[4] June Abbas - Structures for Organizing Knowledge: Exploring Taxonomies, Ontologies, and Other Schema C2010

[5] Kristin Briney - Data Management for Researchers: Organize, maintain and share your data for research success - C2015

[6] NIA - Paul Reip \& Steffi Friendrichs - Forecast of emerging nanotechnologies $\mathrm{C} 2007$

[7] Foresight and indicators for nanotechnology and nanoindustry - by OECD - C2008.

[8] Charles Becht IV - Process Piping - The complete guide to ASME B31.3 - Third Edition - NY USA C2009
[9] Report Merrill Lynch - The Next Small Thing - An Introduction to Nanotechnology - USA C2001

[10] Presentation: Bank of America Merrill Lynch: Taking the Front Office Beyond Traditional Business Intelligence C2015

[11] Manufacturers Standardization Society of the Valve and Fittings Industry (MSS)

[12] Percentiles and percentiles ranks - The technical whitepaper series 3 - C2011

[13] Barry K. Lavine, Clustering and Classification of Analytical Data. Encyclopedia of Analytical Chemistry. Edited by Robert A. Meyers et al. Clarkson University, Potsdam, USA.

[14] ASTM - E2456-06 - Standard Terminology Relating to Nanotechnology - C ASTM International - USA 